\title{
Arbitrary polarization conversion dichroism metasurfaces for all-in-one full Poincaré sphere polarizers
}

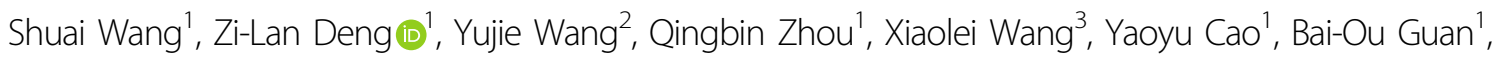 \\ Shumin XiaO (1) ${ }^{2}$ and Xiangping Li (iD)
}

\begin{abstract}
The control of polarization, an essential property of light, is of broad scientific and technological interest. Polarizers are indispensable optical elements for direct polarization generation. However, arbitrary polarization generation, except that of common linear and circular polarization, relies heavily on bulky optical components such as cascading linear polarizers and waveplates. Here, we present an effective strategy for designing all-in-one full Poincaré sphere polarizers based on perfect arbitrary polarization conversion dichroism and implement it in a monolayer all-dielectric metasurface. This strategy allows preferential transmission and conversion of one polarization state located at an arbitrary position on the Poincaré sphere to its handedness-flipped state while completely blocking its orthogonal state. In contrast to previous methods that were limited to only linear or circular polarization, our method manifests perfect dichroism of nearly $100 \%$ in theory and greater than $90 \%$ experimentally for arbitrary polarization states. By leveraging this attractive dichroism, our demonstration of the generation of polarization beams located at an arbitrary position on a Poincaré sphere directly from unpolarized light can substantially extend the scope of meta-optics and dramatically promote state-of-the-art nanophotonic devices.
\end{abstract}

\section{Introduction}

Polarization control is essential for tailoring light-matter interactions and is the foundation for many applications, such as polarization imaging ${ }^{1,2}$, nonlinear optics $^{3,4}$, data storage ${ }^{5,6}$, and information multiplexing ${ }^{7-9}$. A linear polarizer, which is a polarization optical element that filters a specific linear polarization from unpolarized light, plays an important role in both polarization generation and manipulation. However, the generation of

Correspondence: Zi-Lan Deng (zilandeng@jnu.edu.cn) or

Shumin Xiao (shumin.xiao@hit.edu.cn) or Xiangping Li (xiangpingli@jnu.edu.cn)

${ }^{1}$ Guangdong Provincial Key Laboratory of Optical Fiber Sensing and

Communications, Institute of Photonics Technology, Jinan University, 510632 Guangzhou, China

${ }^{2}$ Ministry of Industry and Information Technology Key Lab of Micro-Nano Optoelectronic Information System, Harbin Institute of Technology, 518055

Shenzhen, China

Full list of author information is available at the end of the article

These authors contributed equally: Shuai Wang, Zi-Lan Deng, Yujie Wang arbitrary polarization states other than linear polarization usually requires cascading of multiple optical polarization elements, including both linear polarizers and waveplates based on anisotropic materials ${ }^{10}$ or nanostructures ${ }^{11}$, leading to bulky optical systems that are far from the long-sought miniaturization and integration. Recently, circular polarizers that can directly generate circular polarization from unpolarized light by exploiting the extremely large optical chiral response of 3D chiral nanostructures were proposed ${ }^{12-15}$. To miniaturize these devices, structures with planar optical chirality with only in-plane chiral geometries and preservation of the mirror symmetry in the light propagation direction were proposed $^{16}$, with the advantages of easy fabrication and onchip integration. A variety of planar chiral structures, including fish-scale ${ }^{16}$, asymmetric split ring ${ }^{17}$, gamma$\operatorname{dion}^{18}$, L-shaped ${ }^{19}$, and Z-shaped ${ }^{20}$ structures, have been investigated. In these structures, exotic phenomena such

\section{(c) The Author(s) 2021}

(c) (i) Open Access This article is licensed under a Creative Commons Attribution 4.0 International License, which permits use, sharing, adaptation, distribution and reproduction cc) in any medium or format, as long as you give appropriate credit to the original author(s) and the source, provide a link to the Creative Commons license, and indicate if changes were made. The images or other third party material in this article are included in the article's Creative Commons license, unless indicated otherwise in a credit line to the material. If material is not included in the article's Creative Commons license and your intended use is not permitted by statutory regulation or exceeds the permitted use, you will need to obtain permission directly from the copyright holder. To view a copy of this license, visit http://creativecommons.org/licenses/by/4.0/. 
as circular conversion dichroism (CCD) and asymmetric transmission (AT) occur, in which only one kind of circularly polarized light can be transmitted and converted to its handedness-flipped state, while the orthogonal circular polarization is completely blocked ${ }^{19-21}$.

In parallel, tremendous progress has been made in the area of metasurfaces composed of artificial meta-atoms with tailored phase responses ${ }^{22-36}$, leading to lightweight optical devices such as metalenses ${ }^{37-39}$ and metaholograms $\mathrm{s}^{22,40}$. Polarization control by metasurfaces typically involves anisotropic meta-atom designs to impose different phase retardations on orthogonal linear polarizations, analogous to optical birefringence. By combining the anisotropic dynamic phase with the geometric phase, independent phase retardation can be imposed on any pair of orthogonal polarization states ${ }^{41}$. Elliptical birefringence has also been realized with independent phase manipulation based on elliptical eigenpolarization states, leading to arbitrary polarization conversion under a specified input polarization ${ }^{42}$. In addition, combining the geometric phase with the detour phase can allow simultaneous control of the spatially varying arbitrary polarization and phase profiles, leading to powerful vectorial hologram applications ${ }^{29,34,43}$. However, all of these metasurface polarization optics require an additional polarizer to generate incident beams with well-defined polarization, precluding their applicability in monolithic polarization generators working directly with unpolarized light.

In this paper, we propose an effective approach to achieve full Poincaré sphere polarizers in one step by extending CCD to arbitrary polarization conversion dichroism (APCD) by means of a monolayer metasurface. By using dimerized metamolecules ${ }^{29,34,44,45}$ composed of a pair of birefringent meta-atoms with properly tailored anisotropic phase responses and relative orientation angles, the collective interference of far-field radiation from those meta-atoms can be controlled to generate APCD. This system is able to preferentially transmit one polarization state that can be located at an arbitrary position on a Poincaré sphere ${ }^{46}$ and convert it into transmitted light with flipped handedness while completely blocking the orthogonal polarization state. This APCD metasurface is capable of generating an arbitrarily polarized beam located at an arbitrary position on the Poincaré sphere, irrespective of input polarization, and thus acts as a polarizer that can cover the full Poincaré sphere by design. In practice, we realize such APCD in an all-dielectric metasurface platform in the visible frequency range, manifesting transmissive polarization dichroism (PD) of nearly $100 \%$ in theory and greater than $90 \%$ experimentally. We exploit the perfect PD feature of this system to demonstrate arbitrary polarization, including linear, circular and elliptical polarization, directly from unpolarized light. This all-in-one metasurface polarizer serves as a monolithic arbitrary polarization generator, ultimately promising miniaturized optical devices for integrated nanophotonic systems with substantially reduced complexity.

\section{Results}

We begin with conventional metasurfaces that manipulate polarization states through birefringent meta-atoms, which can impose distinct phase retardations along the fast and slow axes on two orthogonal linear polarizations, as shown in Fig. 1a. Such elementary meta-atoms can be described by the Jones matrix (in terms of electric field) in the linear polarization base as follows ${ }^{41}$ :

$$
\mathbf{J}^{\mathbf{e}}=\mathbf{R}(\theta)\left(\begin{array}{cc}
e^{i \delta_{f}} & 0 \\
0 & e^{i \delta_{s}}
\end{array}\right) \mathbf{R}(-\theta)
$$

where $\delta_{\mathrm{f}}$ and $\delta_{\mathrm{s}}$ represent the phase retardations along the fast and slow axes of the birefringent meta-atoms, respectively, $\theta$ is the orientation angle of the fast axis, and $\mathbf{R}(\theta)=\left(\begin{array}{cc}\cos (\theta) & -\sin (\theta) \\ \sin (\theta) & \cos (\theta)\end{array}\right)$ denotes the rotation matrix. As seen from Eq. 1, the output polarization is heavily dependent upon the incident beam, which restricts its operation to well-defined incident polarizations that typically result from an additional linear polarizer. The proposed APCD metasurface can overcome the aforementioned intrinsic limitations and generate arbitrary polarization states-linear, circular, or elliptical-directly from unpolarized incident light. The metasurface consists of arrays of dimerized metamolecules containing two dielectric birefringent nanopillars, as schematically shown in Fig. 1b. Their far-field interference and collective contributions can be exquisitely tailored by the length, width, and orientation of the nanopillar pairs illuminated by different polarizations, leading to perfect transmissive dichroism for arbitrary orthogonal polarization pairs on demand. In this way, all-in-one metasurface polarizers that allow arbitrary polarization generation covering the full Poincaré sphere directly from unpolarized beams become feasible.

In general, an arbitrary polarization state $\boldsymbol{\alpha}$ can be fully described by two parameters, namely, the main axis angle $\psi$ and the ellipticity angle $X$ (Fig. 2a), which can be represented as a point with coordinates $(2 \psi, 2 \chi)$ on a Poincaré sphere (the red star in Fig. 2a). Its Jones vector can be explicitly written in terms of the parameters $\psi$ and $X$ as follows:

$$
\boldsymbol{\alpha}=\mathbf{R}(\psi)\left(\begin{array}{c}
\cos (\chi) \\
-i \sin (\chi)
\end{array}\right)=\frac{\sqrt{2}}{2} \mathbf{R}\left(\psi-45^{\circ}\right)\left(\begin{array}{c}
e^{i X} \\
e^{-i \chi}
\end{array}\right)
$$




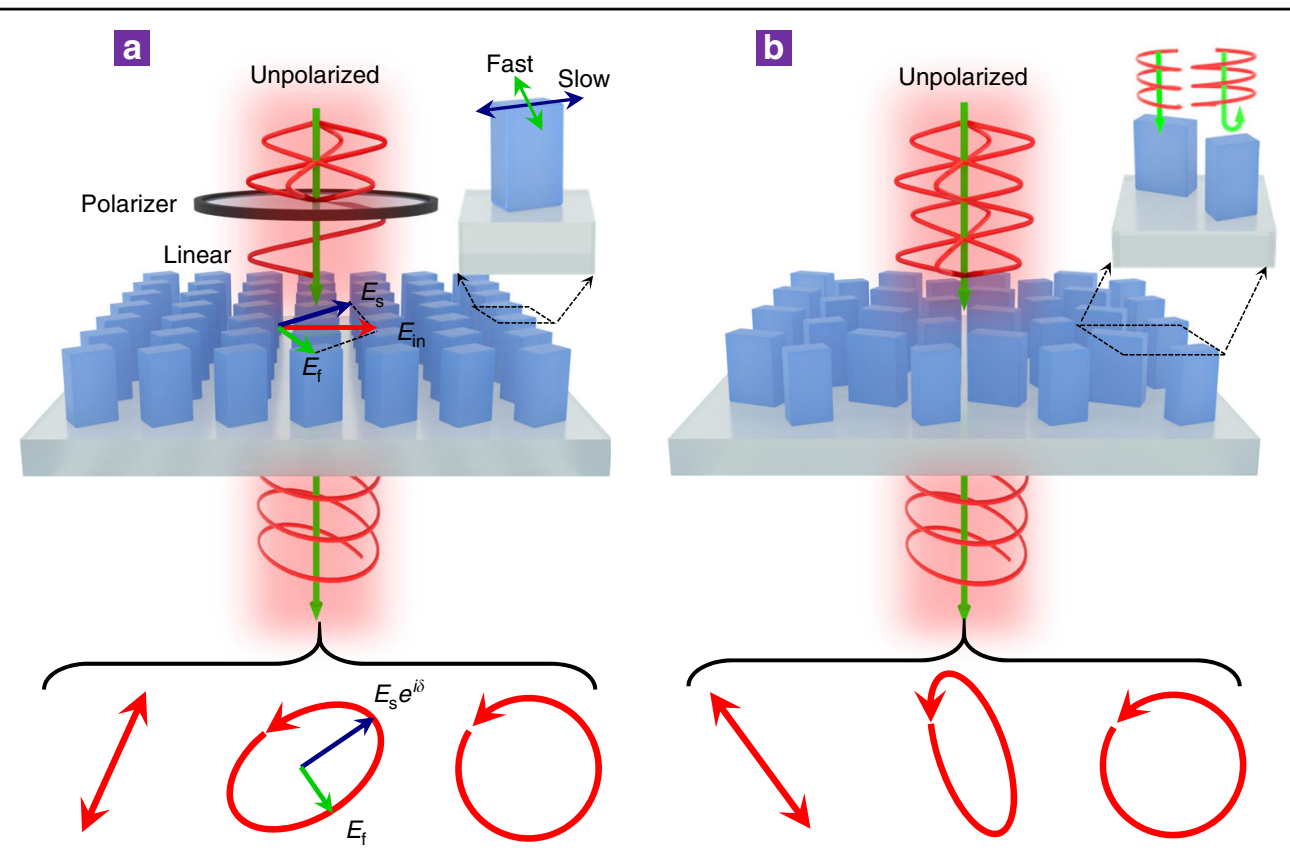

Fig. 1 Comparison of the polarization controls based on the birefringence and dichroism metasurfaces. a Schematic of conventional polarization control based on a cascaded linear polarizer and birefringent single-meta-atom metasurface. The incident beam with well-defined polarization was converted to arbitrary polarization states by imparting different phase retardations on orthogonal linear polarizations along the fast and slow axes of the birefringent meta-atom. $\mathbf{b}$ Schematic of the proposed all-in-one polarizer that can function at an arbitrary position on the Poincaré sphere by design based on a dielectric metasurface composed of dimerized nanopillars, which can directly operate with unpolarized incident light and generate arbitrary polarization states, including linear, elliptical and circular polarizations, regardless of the incident polarization state.

Its orthogonal polarization state $\boldsymbol{\beta}$ is located at the inversion symmetry point (the blue star in Fig. 2a) with coordinates $\left(2\left(\psi-90^{\circ}\right),-2 \chi\right)$ and the following Jones vector:

$$
\boldsymbol{\beta}=\mathbf{R}\left(\psi-90^{\circ}\right)\left(\begin{array}{c}
\cos (X) \\
i \sin (\chi)
\end{array}\right)=\frac{\sqrt{2}}{2} \mathbf{R}\left(\psi-45^{\circ}\right)\left(\begin{array}{c}
e^{i x} \\
-e^{-i X}
\end{array}\right)
$$

As we can see, $\alpha$ and $\beta$ can be written as the product of a rotation matrix with the same angle $\psi-45^{\circ}$ and a Jones vector composed of only the parameter $X$; therefore, we can define a local x'oy' coordinate system that is rotated by $\psi$ $-45^{\circ}$ with respect to the global xoy coordinate system (Fig. 2b) to simplify the deduction of the required Jones matrix for APCD (Supplementary Note 1). In addition, the handedness-flipped states $\boldsymbol{\alpha}^{*}$ and $\boldsymbol{\beta}^{*}$ (where * denotes the complex conjugate operator) are located at mirror symmetry points with respect to the equatorial plane, as denoted by the red and blue dots, respectively, in Fig. 2a. To formulate the Jones matrix $\mathbf{J}$ for APCD that allows transmission and conversion of the polarization state $\boldsymbol{\alpha}$ to its handedness-flipped polarization $\boldsymbol{\alpha}^{*}$ and completely blocks $\boldsymbol{\beta}$, it is convenient to define another Jones matrix $\mathbf{J}^{\#}$ connecting the input and output polarization states defined in the $(\boldsymbol{\alpha}, \boldsymbol{\beta})$ base and $\left(\boldsymbol{\alpha}^{*}, \boldsymbol{\beta}^{*}\right)$ base, respectively. By applying a series of base transformations from a linear polarization base in the global xoy system to a linear polarization base in the local x'oy' system and finally to an arbitrary polarization base, we can obtain $\mathbf{J}^{\#}$ in terms of the parameters $\psi$ and $X$ as follows (Supplementary Note 1):

$\mathbf{J}=\left(\begin{array}{cc}t_{\alpha^{*} \alpha} & t_{\beta^{*} \alpha} \\ t_{\alpha^{*} \beta} & t_{\beta^{*} \beta}\end{array}\right)=\left(\begin{array}{cc}e^{-i x} & e^{-i x} \\ e^{i x} & -e^{i x}\end{array}\right)^{-1} \mathbf{R}\left(45^{\circ}-\psi\right) \mathbf{J}\left(\psi-45^{\circ}\right)\left(\begin{array}{cc}e^{i x} & e^{i x} \\ e^{-i x} & -e^{-i x}\end{array}\right)$

where the matrix elements $t_{j i}\left(i=\alpha, \beta ; j=\alpha^{*}, \beta^{*}\right)$ represent the conversion coefficients from polarization state $\mathbf{i}$ to state $\mathbf{j}$. Perfect APCD requires $t_{\alpha^{* \prime} \alpha}=1$, and $\mathrm{t}_{\beta^{* \alpha} \alpha}=\mathrm{t}_{\alpha^{*} \beta}=\mathrm{t}_{\beta^{*} \beta}=0$. Upon substituting those conditions into Eq. 4, we can obtain the Jones matrix $\mathbf{J}$ in the xoy coordinate as follows (Supplementary Note 1):

$$
\mathbf{J}=\frac{1}{2} \mathbf{R}\left(\psi-45^{\circ}\right)\left(\begin{array}{cc}
e^{-2 i x} & 1 \\
1 & e^{2 i x}
\end{array}\right) \mathbf{R}\left(45^{\circ}-\psi\right)
$$

To achieve its physical implementation with explicit birefringent meta-atoms as described by Eq. 1, Eq. 5 can 

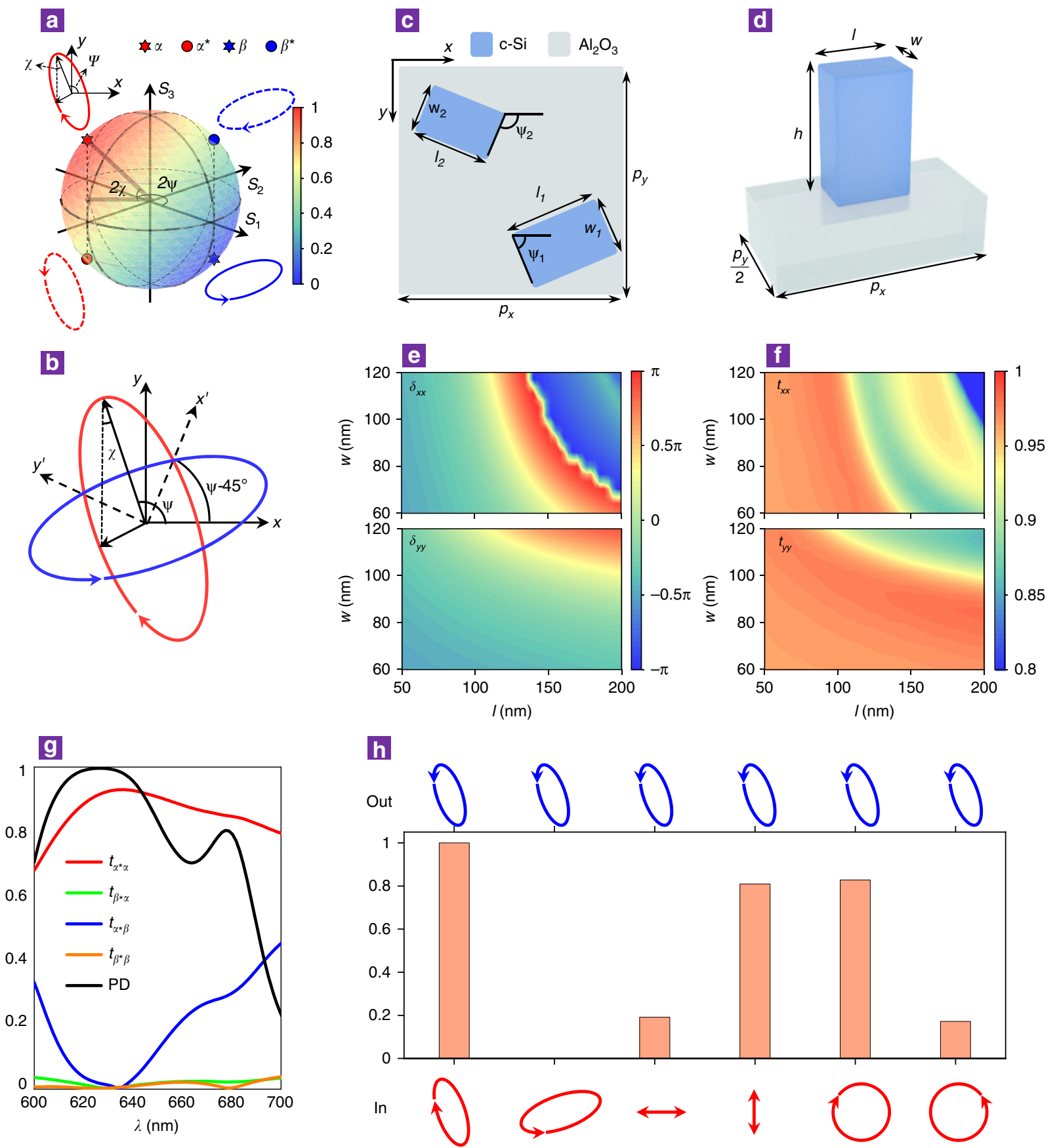

Fig. 2 Metasurface design for APCD. a Poincaré sphere representation of an arbitrary orthogonal polarization pair ( $\boldsymbol{\alpha}, \boldsymbol{\beta}$ ) (solid red and blue ellipses) and its handedness-flipped pair $\left(\mathbf{a}^{*}, \boldsymbol{\beta}^{*}\right)$ (dashed red and blue ellipses). b Defined local coordinate system $x^{\prime} \circ y^{\prime}$ (dashed lines) that is rotated by $\psi$-45 with respect to the global xoy coordinate, in which the axes pass through cross points of the polarization ellipses of $\mathbf{a}$ and $\boldsymbol{\beta}$. c Schematic of metamolecules on the metasurface consisting of asymmetrically dimerized nanopillars. The periods are $p_{x}=p_{y}=340 \mathrm{~nm}$, and the orientation angles and positions of the two nanopillars are $\psi_{1}=\psi-45^{\circ}$ and $\psi_{2}=\psi$ and $\left(3 p_{x} / 4,3 p_{y} / 4\right)$ and $\left(p_{x} / 4, p_{y} / 4\right)$, respectively, where $\psi$ is the main axis angle of the modulated polarization ellipse. $\mathbf{d}$ Single nanopillar unit cell for determining phase retardations along its length and width, as shown in e. The height of the nanopillar was fixed at $300 \mathrm{~nm}$. e Phase retardations and $\mathbf{f}$ transmission coefficients upon varying the length / and width $w$ of the nanopillar at a wavelength of $633 \mathrm{~nm}$ for the $x$-polarized (upper panels) and $y$-polarized (lower panels) incident light. $\mathbf{g}$ Polarization conversion coefficients and PD spectrum calculated with the optimized parameters $\left(I_{1}=130 \mathrm{~nm}, w_{1}=70 \mathrm{~nm} ; I_{2}=150 \mathrm{~nm}, w_{2}=85 \mathrm{~nm}\right)$ at a wavelength of $633 \mathrm{~nm}$, which yields $t_{\alpha^{*} \alpha}=0.93$ and $t_{\beta^{*} \alpha}=t_{\alpha^{*} \beta}=t_{\beta^{*} \beta} \approx 0$. $\mathbf{h}$, Transmitted polarization states (blue ellipses) when the metasurface was illuminated by a variety of different incident polarization states (red curves). The histogram indicates the transmittance, which varied with the incident polarization, while the shape of the blue ellipse was the same as that of the designed polarization state $\boldsymbol{a}^{*}$. 
be decomposed as the superposition of two linear birefringent waveplates (Supplementary Note 1), as follows:

$$
\mathbf{J}=\frac{1}{2} \mathbf{R}\left(\psi-45^{\circ}\right)\left(\begin{array}{cc}
e^{-2 i x} & 0 \\
0 & e^{2 i x}
\end{array}\right) \mathbf{R}\left(45^{\circ}-\psi\right)+\frac{1}{2} \mathbf{R}(\psi)\left(\begin{array}{cc}
1 & 0 \\
0 & -1
\end{array}\right) \mathbf{R}(-\psi)
$$

The first term in Eq. 6 can be physically realized by a birefringent meta-atom that has an orientation angle of $\psi-45^{\circ}$ and imposes phase shifts of $-2 x$ and $2 x$ along its fast and slow axes, respectively. The second term has phase retardations of 0 and $\pi$ along the fast and slow axes, respectively, and an orientation angle of $\psi$.

In the practical metasurface design, we use high-index alldielectric nanopillars composed of crystalline silicon (c-Si) on top of an $\mathrm{Al}_{2} \mathrm{O}_{3}$ substrate as our building blocks (Fig. 2c). Once the polarization parameters $(\psi, \chi)$ for dichroism are determined, the two nanopillars with properly tailored lengths $l$ and widths $w$ will be selected for meta-molecule designs. To generate the lookup table for birefringent metaatoms with optimal geometries to satisfy Eq. 6, we first examine the amplitude and phase of light transmitted from a metasurface composed of periodic arrays of single nanopillars, as shown in Fig. 2d. The amplitude and phase responses calculated from periodic arrays are used to approximate those meta-atoms in Eq. 6. The imposed phase retardations $\left(\delta_{x x}\right.$ and $\left.\delta_{y y}\right)$ and transmission coefficients $\left(t_{x x}\right.$ and $t_{y y}$ ) for the $x$ - and $y$-polarized beams are determined through numerical simulations by varying the geometric dimensions of the nanopillars, as depicted in Fig. 2e, f. Distinct phase retardations along the fast and slow axes of the nanopillars can be flexibly configured by properly designing their lengths and widths while maintaining transmission coefficients greater than $90 \%$.

Without loss of generality, we first designed a metasurface that allows preferential transmission of the polarization state $\boldsymbol{\alpha}\left(\psi=112.5^{\circ}, X=22.5^{\circ}\right)$ and completely rejects its orthogonal state $\boldsymbol{\beta}\left(\psi-90^{\circ}=22.5^{\circ},-X=\right.$ $-22.5^{\circ}$ ). Based on the above analysis, optimal geometric parameters of $l_{1}=130 \mathrm{~nm}, w_{1}=70 \mathrm{~nm}, l_{2}=150 \mathrm{~nm}, w_{2}$ $=85 \mathrm{~nm}$ and $h=300 \mathrm{~nm}$ were chosen for the elliptical polarization conversion dichroism. The arrangement of the meta-atoms is shown in Fig. 2c, where the centre positions of the two nanopillars were optimized to $\left(3 p_{x} / 4\right.$, $\left.3 p_{y} / 4\right)$ and $\left(p_{x} / 4, p_{y} / 4\right)$ (Supplementary Note 1 and Fig. S2). To characterize the dichroism performance, we defined the transmissive PD as the relative difference in transmittances of polarization states $\boldsymbol{\alpha}$ and $\boldsymbol{\beta}$,

$$
\mathrm{PD}=\frac{T_{\alpha}-T_{\beta}}{T_{\alpha}+T_{\beta}}
$$

The calculated polarization conversion coefficients (defined as matrix elements of $\mathbf{J}^{\#}$ in Eq. 4) as well as the PD spectrum are plotted in Fig. $2 \mathrm{~g}$ as a function of wavelength. Here, the polarization conversion coefficient of $t_{\alpha^{\prime \prime} \alpha}$ is 0.93 , and the other three coefficients are completely suppressed at a wavelength of $633 \mathrm{~nm}$, resulting in a peak value of PD close to unity (black curve in Fig. 2g), indicating perfect polarization conversion dichroism for the orthogonal elliptical polarization pair $(\boldsymbol{\alpha}, \boldsymbol{\beta})$. This metasurface serves as a perfect arbitrary polarizer that allows a specific elliptical polarization to pass through, regardless of the polarization state of the incident light. As shown in Fig. $2 \mathrm{~h}$, for incidence light with various input polarizations (denoted by red ellipses, lines and circles), the polarization of the transmitted light (blue ellipses) maintains the same state $\boldsymbol{\alpha}^{*}\left(\psi=112.5^{\circ}\right.$, $-\chi=-22.5^{\circ}$ ), while the transmittance is determined by the projection of the incident polarization states on $\alpha$ in the polarization base vector $(\boldsymbol{\alpha}, \boldsymbol{\beta})$, as shown in the histograms.

The freedom provided by the proposed metasurface allows full Poincaré sphere polarization control. To demonstrate the viability and versatility, we experimentally fabricated three dichroic metasurfaces to achieve three kinds of polarization: elliptical $\left(\psi=112.5^{\circ}, \chi=\right.$ $\left.22.5^{\circ}\right)$, linear $\left(\psi=112.5^{\circ}, \chi=0\right)$, and circular $\left(\psi=112.5^{\circ}\right.$, $\left.X=45^{\circ}\right)$. The corresponding scanning electron microscopy (SEM) images are shown in Fig. 3a-c. Figure 3d, e shows the simulated and experimentally measured transmittance of the elliptical polarization conversion dichroism metasurface illuminated by all possible polarization states marked in the Poincaré sphere. The incident polarization states with maximum and minimum transmittance values are plotted as the red and blue stars and the red and blue ellipses, respectively, which are consistent with the corresponding elliptical polarization states $\boldsymbol{\alpha}$ and $\boldsymbol{\beta}$ that are allowed and blocked by the metasurface design. The dashed red ellipse indicates the polarization state $\boldsymbol{\alpha}^{*}$ of the output beam with flipped handedness and almost identical ellipticity as the incident polarization $\boldsymbol{\alpha}$, which unambiguously verifies the elliptical polarization conversion dichroism. We note that the polarization states in the experiment deviated slightly from those of the simulation, which can be attributed to fabrication imperfection. The PD spectrum shows a peak close to unity in the simulation (Fig. 3j) and greater than 95\% in the experiment (Fig. 3k). Benefitting from such high dichroism performance, it can generate an elliptical polarization state $\boldsymbol{\alpha}^{*}$ independent of the incident polarization, which is presented in Supplementary Note 2 and Fig. S3.

Similarly, the transmittance of the linear and circular polarization conversion dichroism metasurfaces under illumination by all possible polarization states covering the Poincaré sphere is shown in Fig. $3 \mathrm{f}-\mathrm{g}$ and Fig. $3 \mathrm{~h}-\mathrm{i}$, respectively. The incident polarization states with maximum and minimum transmittances are distributed on the opposite site of the Poincaré sphere, representing two 


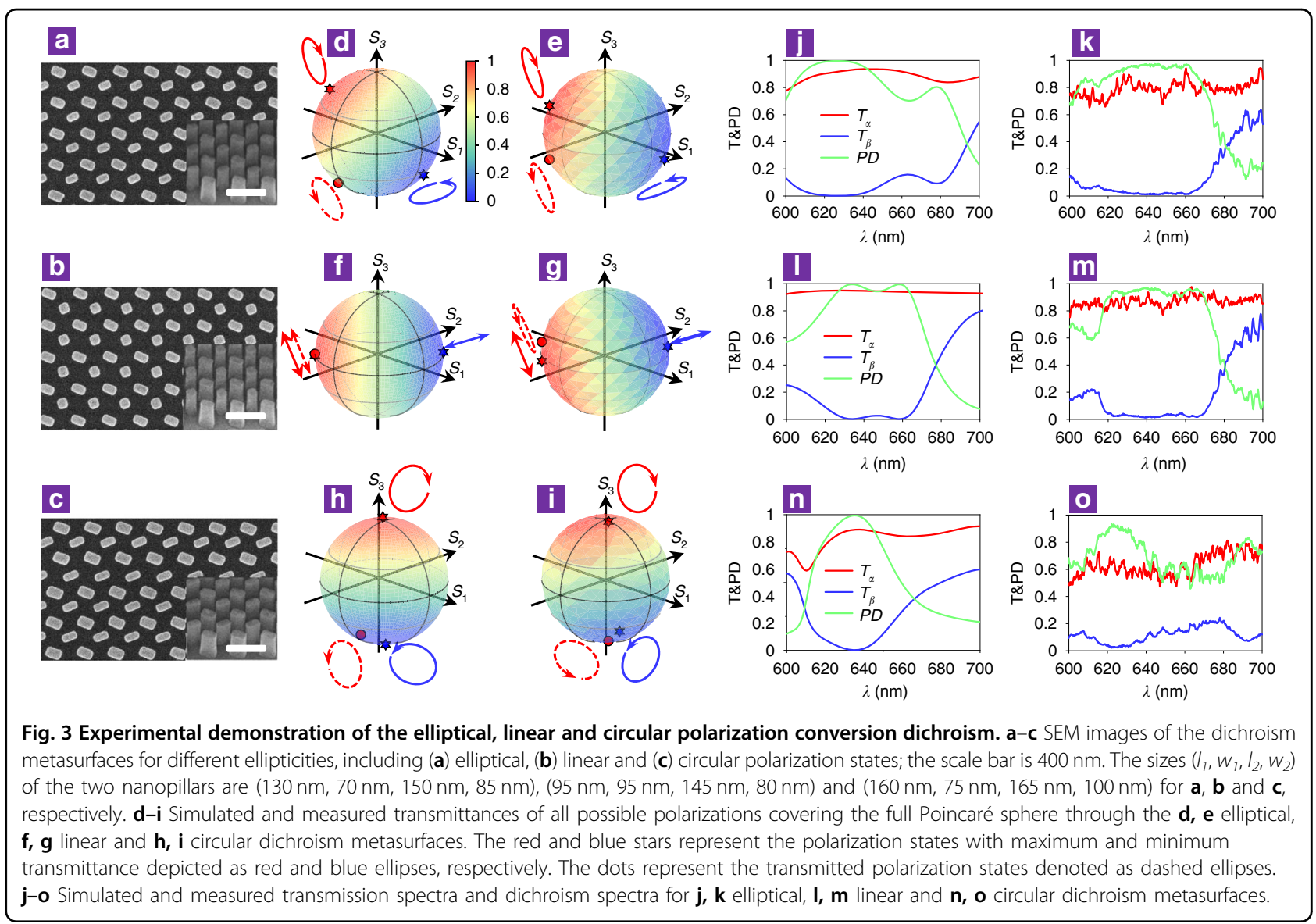

pairs of orthogonal polarizations, namely, linear and circular polarization states, respectively. Likewise, the PDs are close to unity in the simulation and greater than $95 \%$ for the linear polarization (Fig. 3l, m) and $90 \%$ for the circular polarization (Fig. $3 \mathrm{n}-\mathrm{o}$ ) in the experiment. Notably, the APCD metasurfaces also support AT phenomena beyond linear and circular polarization ${ }^{16,47}$, as demonstrated in Supplementary Note 3 and Fig. S4. The allowed and prevented polarization states of the APCD metasurface can be switched by simply swapping the length and width parameters of either of the two nanopillars, as shown in Supplementary Note 4 and Fig. S5. Beyond the three abovementioned representative polarization conversion dichroisms, APCD designs covering the full Poincaré sphere can be implemented by means of the procedure introduced in Supplementary Note 5 and Figs. S6, S7. First, we can achieve polarization conversion dichroism along the latitude lines of the Poincaré sphere with different ellipticities $X$ by sweeping nanopillar dimensions. Then, we can simply rotate the entire metasurface to realize another polarization conversion dichroism along the longitude lines on the Poincare sphere, which represent polarization states with an identical ellipticity $x$ but different $\psi$. By combining the above two steps, APCD covering the full Poincaré sphere can be obtained.

As a proof of principle, we exploit APCD to demonstrate direct polarization generation from unpolarized light. Figure 4a shows the experimental configuration, where an LED is employed as the unpolarized light source. The polarization state of the transmitted beam can be obtained by measuring the Stokes parameters. Figure $4 \mathrm{~b}$ shows the measured Stokes parameters and the degree of polarization ( $D o P$, defined as $\sqrt{S_{1}^{2}+S_{2}^{2}+S_{3}^{2}}$ ) directly from the LED beam source. Indeed, the polarization state directly from the LED source is undefined. After passing through the monolithic APCD metasurfaces, the measured $D o P s$ are close to unity, indicating a well-defined polarization state centred at a wavelength of $633 \mathrm{~nm}$ (Fig. 4c-e). The output polarizations can be obtained by analysing the Stokes parameters derived from the measured intensities of four linear polarizations polarized along $x, y, 45^{\circ}$, and $135^{\circ}$ and two circular polarizations in the transmitted beams, as shown in the histograms in Fig. 4c-e, which are depicted by the polarization ellipses determined by the main axis angle $\psi$ and the ellipticity angle $X$. The measured output 


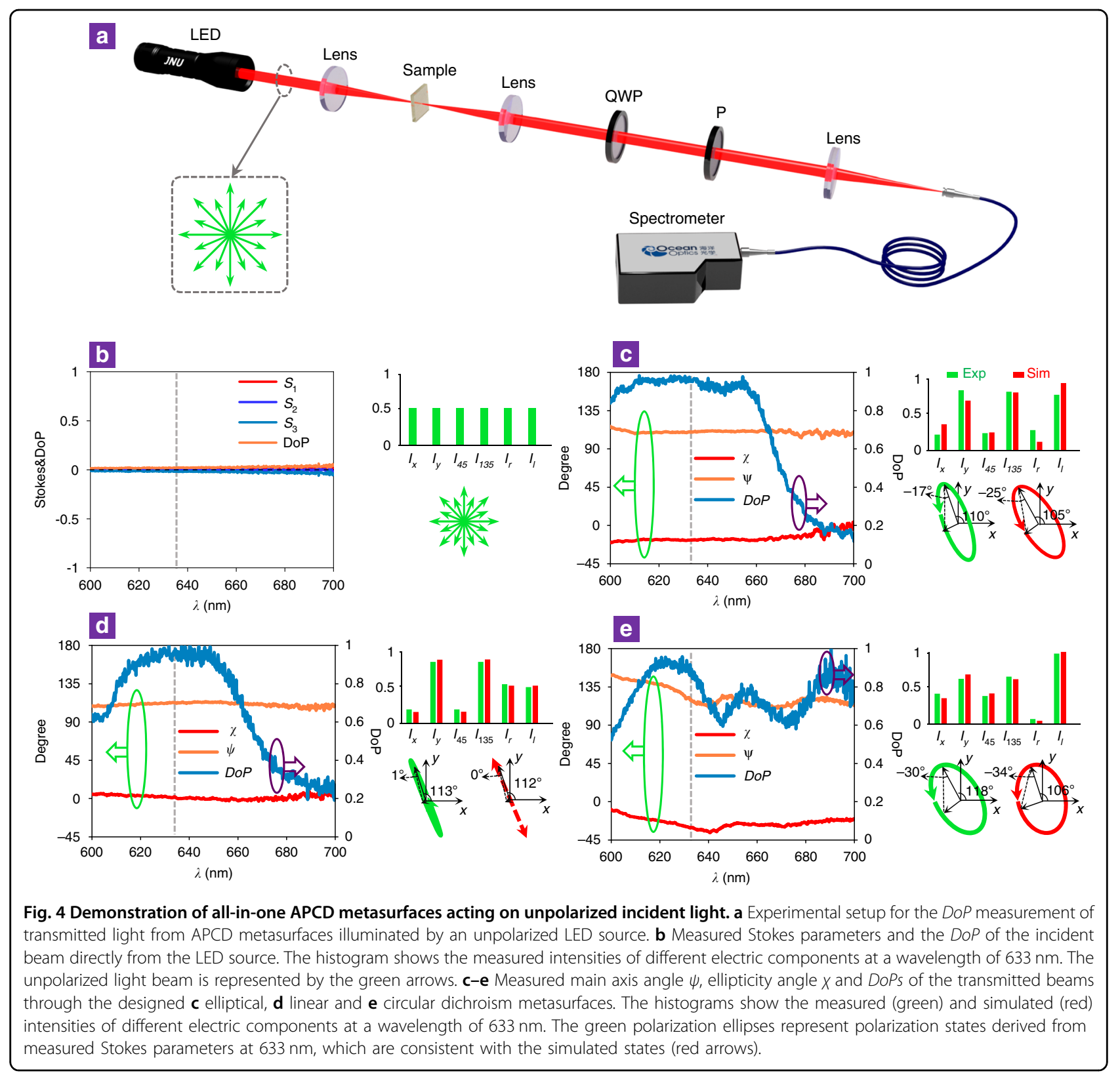

polarization states (green) show reasonable congruence with the simulated state (red). Although the main axis angle $\psi$ and the ellipticity angle $X$ of the output polarization states at other wavelengths exhibit deviate slightly from the ideal case at $633 \mathrm{~nm}$, the DoPs are measured to maintain a value above 0.9 over a bandwidth for linear, elliptical and circular dichroism metasurfaces, promising high-performance polarizers.

\section{Discussion}

In this paper, we have proposed a general design for alldielectric metasurfaces that can realize perfect APCD and demonstrated their applicability for all-in-one polarizers that function at an arbitrary position on the Poincaré sphere by design. The proposed APCD metasurfaces are composed of asymmetrically dimerized birefringent dielectric nanopillars with certain intersection angles, enabling us to exquisitely configure their far-field interference for perfect dichroism close to $100 \%$ in the simulation and greater than $90 \%$ in experiments for arbitrary polarizations, including linear, circular or elliptical polarizations. The advanced features and design flexibility of the proposed metasurface offer the potential for the development of all-in-one metasurface polarizers directly operating with unpolarized beams for full Poincaré sphere polarization manipulations, which can dramatically encourage 
work on state-of-the-art planar optics and extensively promote integrated devices based on meta-optics.

\section{Materials and methods}

\section{Theoretical design of the APCD metasurface}

Numerical simulations were carried out by using the finite element method (FEM). First, polarization conversion coefficients and phase retardations under illumination by linearly polarized beams were calculated, with periodic boundary conditions applied in both the $x$ and $y$ directions. Second, the Jones matrix was obtained by analysing the results from the FEM simulation. The transmittance under all polarization states covering the full Poincare sphere was obtained by multiplying the polarization state of the incident light by the Jones matrix, where incident beams with different polarizations were superposed by the two orthogonal linear-polarized base vectors.

\section{Experimental fabrication of an APCD metasurface}

The metasurface was fabricated on commercially available $300-\mathrm{nm}$ thick c-Si (100) epitaxially grown on a sapphire substrate (from UniversityWafer, Inc.). The structure was patterned on a positive resist (PMMA A2) using an E-beam writer (Raith E-line, $30 \mathrm{kV}$ ). After developing the resist, we transferred the pattern from the resist onto a chromium film with a thickness of $25 \mathrm{~nm}$. Then, the silicon layer was etched by inductively coupled plasma etching (PlasmaPro System 100ICP180) using chromium as a hard mask. The remaining $\mathrm{Cr}$ was removed with a chromium etchant.

\section{Characterization of the APCD metasurface}

A supercontinuum laser (Fianium-WL-SC480) was employed as the broadband light source for measurement of the transmission spectra under a specific polarization incidence, while an LED was used for unpolarized incident light. Incident light with a specific polarization located at an arbitrary position on the Poincaré sphere was generated by cascading a broadband linear polarizer and quarter waveplate from the supercontinuum laser. Then, the incident light was focused on the sample by a lens with a focal length of $10 \mathrm{~cm}$. The transmittance and Stokes parameters were determined by means of an Ocean spectrometer (USB4000).

\section{Acknowledgements}

This work was supported by the National Key R\&D Program of China (2018YFB1 107200), the National Natural Science Foundation of China (NSFC) (Grants 62075084, 61522504, 61420106014, 11734012, and 11574218), the Guangdong Provincial Innovation and Entrepreneurship Project (Grant 2016ZT06D081), the Guangdong Basic and Applied Basic Research Foundation (2020A1515010615), the Fundamental Research Funds for the Central Universities (21620415) and the China Scholarship Council (201906785011).

\section{Author details}

${ }^{1}$ Guangdong Provincial Key Laboratory of Optical Fiber Sensing and Communications, Institute of Photonics Technology, Jinan University, 510632
Guangzhou, China. ${ }^{2}$ Ministry of Industry and Information Technology Key Lab of Micro-Nano Optoelectronic Information System, Harbin Institute of Technology, 518055 Shenzhen, China. Institute of Modern Optics, Nankai University, 300350 Tianjin, China

\section{Author contributions}

S.W. and Z.-L.D. conceived the idea. S.W., Z.-L.D. and X.L. designed the experiments. S.W. and Z.-L.D. carried out the theoretical analysis and the design and simulation of the metasurfaces. Y.W. and S.X. fabricated the samples. S.W. and Q.Z. performed the measurements. Z.-L.D. and X.L. supervised the project. S.W., Z.-L.D., S.X. and X.L. analysed the data and wrote the manuscript. All authors contributed to the discussion of the manuscript.

\section{Conflict of interest}

The authors declare that they have no conflict of interest.

Supplementary information The online version contains supplementary material available at https://doi.org/10.1038/s41377-021-00468-y.

Received: 19 July 2020 Revised: 1 January 2021 Accepted: 7 January 2021 Published online: 27 January 2021

\section{References}

1. Rubin, N. A. et al. Matrix Fourier optics enables a compact full-Stokes polarization camera. Science 365, 43 (2019).

2. Hu, H., Gan, Q. \& Zhan, Q. Generation of a nondiffracting superchiral optical needle for circular dichroism imaging of sparse subdiffraction objects. Phys. Rev. Lett. 122, 223901 (2019).

3. Li, G. et al. Continuous control of the nonlinearity phase for harmonic generations. Nat. Mater. 14, 607-612 (2015).

4. Segal, N., Keren-Zur, S., Hendler, N. \& Ellenbogen, T. Controlling light with metamaterial-based nonlinear photonic crystals. Nat. Photonics 9, 180-184 (2015).

5. Zijlstra, P., Chon, J. W. \& Gu, M. Five-dimensional optical recording mediated by surface plasmons in gold nanorods. Nature 459, 410-413 (2009).

6. Li, X., Lan, T.-H., Tien, C.-H. \& Gu, M. Three-dimensional orientation-unlimited polarization encryption by a single optically configured vectorial beam. Nat. Commun. 3, 1-6 (2012).

7. Chen, Z.-Y. et al. Use of polarization freedom beyond polarization-division multiplexing to support high-speed and spectral-efficient data transmission. Light Sci. Appl. 6, e16207-e16207 (2017).

8. Wang, J. et al. Terabit free-space data transmission employing orbital angular momentum multiplexing. Nat. Photonics 6, 488 (2012).

9. Zhang, F. et al. All-dielectric metasurfaces for simultaneous giant circular asymmetric transmission and wavefront shaping based on asymmetric photonic spin-orbit Interactions. Adv. Funct. Mater. 27, 1704295 (2017).

10. Born, M. \& Wolf, E. Principles of optics: electromagnetic theory of propagation, interference and diffraction of light 7th edn. (Cambridge University Press, Cambridge, 1999)

11. Noda, S., Yokoyama, M., Imada, M., Chutinan, A. \& Mochizuki, M. Polarization mode control of two-dimensional photonic crystal laser by unit cell structure design. Science 293, 1123-1125 (2001).

12. Gansel, J. K. et al. Gold helix photonic metamaterial as broadband circular polarizer. Science 325, 1513-1515 (2009).

13. Zhao, Y., Belkin, M. A. \& Alù, A. Twisted optical metamaterials for planarized ultrathin broadband circular polarizers. Nat. Commun. 3, 870 (2012).

14. Turner, M. D. et al. Miniature chiral beamsplitter based on gyroid photonic crystals. Nat. Photonics 7, 801-805 (2013).

15. Pfeiffer, C., Zhang, C., Ray, V., Guo, L. J. \& Grbic, A. High performance bianisotropic metasurfaces: asymmetric transmission of light. Phys. Rev. Lett. 113, 023902 (2014).

16. Fedotov, V. A. et al. Asymmetric propagation of electromagnetic waves through a planar chiral structure. Phys. Rev. Lett. 97, 167401 (2006).

17. Plum, E., Fedotov, V. A. \& Zheludev, N. I. Planar metamaterial with transmission and reflection that depend on the direction of incidence. Appl. Phys. Lett. 94, 131901 (2009).

18. Zhu, A. Y. et al. Giant intrinsic chiro-optical activity in planar dielectric nanostructures. Light Sci. Appl. 7, 17158-17158 (2018). 
19. Ye, W. et al. Large chiroptical effects in planar chiral metamaterials. Phys. Rev. Appl. 7, 054003 (2017).

20. $\mathrm{Ma}$, Z. et al. All-dielectric planar chiral metasurface with gradient geometric phase. Opt. Express 26, 6067-6078 (2018).

21. Kenney, M. et al. Pancharatnam-berry phase induced spin-selective transmission in herringbone dielectric metamaterials. Adv. Mater. 28, 9567-9572 (2016).

22. Zheng, G. et al. Metasurface holograms reaching $80 \%$ efficiency. Nat. Nanotechnol. 10, 308-312 (2015).

23. $\mathrm{Yu}, \mathrm{N}$. et al. Light propagation with phase discontinuities: generalized laws of reflection and refraction. Science 334, 333-337 (2011).

24. Huang, L. et al. Dispersionless phase discontinuities for controlling light propagation. Nano Lett. 12, 5750-5755 (2012).

25. Deng, Z.-L. et al. Facile metagrating holograms with broadband and extreme angle tolerance. Light Sci. Appl. 7, 1-8 (2018).

26. Overvig, A. C. et al. Dielectric metasurfaces for complete and independent control of the optical amplitude and phase. Light Sci. Appl. 8, 92 (2019).

27. Lee, G.Y. et al. Complete amplitude and phase control of light using broadband holographic metasurfaces. Nanoscale 10, 4237-4245 (2018).

28. Arbabi, A., Horie, Y., Bagheri, M. \& Faraon, A. Dielectric metasurfaces for complete control of phase and polarization with subwavelength spatial resolution and high transmission. Nat. Nanotechnol. 10, 937-943 (2015).

29. Deng, Z.-L. et al. Diatomic metasurface for vectorial holography. Nano Lett. 18, 2885-2892 (2018)

30. $\mathrm{Yu}, \mathrm{N}$. et al. A broadband, background-free quarter-wave plate based on plasmonic metasurfaces. Nano Lett. 12, 6328-6333 (2012).

31. Sun, W., He, Q., Hao, J. \& Zhou, L. A transparent metamaterial to manipulate electromagnetic wave polarizations. Opt. Lett. 36, 927-929 (2011).

32. Huang, K. et al. Photon-nanosieve for ultrabroadband and large-angle-of-view holograms. Laser Photonics Rev. 11, 1700025 (2017).

33. Huang, $\mathrm{K}$. et al. Ultrahigh-capacity non-periodic photon sieves operating in visible light. Nat. Commun. 6, 7059 (2015).
34. Deng, Z.-L. et al. Full-color complex-amplitude vectorial holograms based on multi-freedom metasurfaces. Adv. Funct. Mater. 30, 1910610 (2020).

35. Wang, S. et al. Diatomic metasurface based broadband J-plate for arbitrary spin-to-orbital conversion. J. Phys. D. Appl. Phys. 52, 324002 (2019).

36. Ma, X., Pu, M., Li, X., Guo, Y. \& Luo, X. All-metallic wide-angle metasurfaces for multifunctional polarization manipulation. Opto Electron Adv. 2, 180023 (2019).

37. Khorasaninejad, M. \& Capasso, F. Metalenses: versatile multifunctional photonic components. Science 358, eaam8100 (2017)

38. Lin, R. J. et al. Achromatic metalens array for full-colour light-field imaging. Nat Nanotechnol. 14, 227-231 (2019).

39. Chen, W. T. et al. A broadband achromatic metalens for focusing and imaging in the visible. Nat. Nanotechnol. 13, 220-226 (2018).

40. Deng, Z.-L. \& Li, G. Metasurface optical holography. Mater. Today Phys. 3, 16-32 (2017).

41. Mueller, J. B., Rubin, N. A., Devlin, R. C., Groever, B. \& Capasso, F. Metasurface polarization optics: independent phase control of arbitrary orthogonal states of polarization. Phys. Rev. Lett. 118, 113901 (2017).

42. Shi, Z. et al. Continuous angle-tunable birefringence with freeform metasurfaces for arbitrary polarization conversion. Sci. Adv. 6, eaba3367 (2020).

43. Song, Q. et al. Ptychography retrieval of fully polarized holograms from geometric-phase metasurfaces. Nat. Commun. 11, 2651 (2020).

44. Overvig, A. C., Shrestha, S. \& Yu, N. Dimerized high contrast gratings. Nanophotonics 7, 1157 (2018).

45. Bao, Y. et al. Full-colour nanoprint-hologram synchronous metasurface with arbitrary hue-saturation-brightness control. Light Sci. Appl. 8, 95 (2019).

46. Milione, G., Sztul, H. I., Nolan, D. A. \& Alfano, R. R. Higher-order Poincaré sphere, stokes parameters, and the angular momentum of light. Phys. Rev. Lett. 107, 053601 (2011).

47. Menzel, C., Rockstuhl, C. \& Lederer, F. Advanced Jones calculus for the classification of periodic metamaterials. Phys. Rev. A 82, 053811 (2010). 